\title{
Mesenchymal stem cell-derived exosomes regulate the polarization and inflammatory response of macrophages via miR- 21-5p to promote repair after myocardial reperfusion injury
}

\author{
Dafu Shen ${ }^{1}$, Zhiwei $\mathrm{He}^{2}$ \\ ${ }^{1}$ Department of Cardiovascular Surgery, Renji Hospital, Shanghai Jiao Tong University School of Medicine, Shanghai, China; ${ }^{2}$ Department of \\ General Surgery, Shanghai Post and Telecommunication Hospital, Shanghai, China \\ Contributions: (I) Conception and design: D Shen; (II) Administrative support: Z He; (III) Provision of study materials or patients: D Shen; (IV) \\ Collection and assembly of data: Both authors; (V) Data analysis and interpretation: Both authors; (VI) Manuscript writing: Both authors; (VII) Final \\ approval of manuscript: Both authors. \\ Correspondence to: Zhiwei He. Department of General Surgery, Shanghai Post and Telecommunication Hospital, 666 Changle Road, Shanghai, China. \\ Email: shydyy6613@163.com.
}

Background: Myocardial ischemia-reperfusion injury is a type of myocardial ischemia that has a significant impact on patients' health. We aimed to explore the protective effect of mesenchymal stem cell-derived exosomes (MSC-EXOs) on myocardial ischemia-reperfusion injury and their specific mechanism.

Methods: The effects of MSC-EXOs on myocardial ischemia-reperfusion injury were recorded. An enzyme-linked immunosorbent assay (ELISA) was used to determine the levels of IL-6 and IL-10 in mouse myocardial tissue or culture supernatant. Co-cultured MSC-EXOs and RAW264.7 cells were used to study the effect of MSC-EXOs on the polarization of macrophages at the cellular level. The ratio of M1 and M2 macrophages were detected by flow cytometry, and RT-qPCR detected the mRNA expression levels of corresponding markers. After transfection with miR-21-5p inhibitors or mimics, flow cytometry and RTqPCR experiments were performed to explore the specific role of MSC-EXOs in macrophage polarization.

Results: After injection of MSC-EXOs, the mRNA expression of M1 macrophage markers (iNOS, IL-1 $\beta$, IL-6, and TNF $\alpha$ ) in the myocardial tissue of model mice was significantly reduced $(\mathrm{P}<0.05)$, and the mRNA expression of M2 macrophage markers was significantly increased $(\mathrm{P}<0.05)$. The injection also reduced the inflammation response in the model mice $(\mathrm{P}<0.05)$. In the in vitro experiment, lipopolysaccharide (LPS) induced the inflammatory microenvironment. After MSC-EXOs were fixed in the cytoplasm of RAW264.7 cells, the level of IL-6 in the culture supernatant decreased $(\mathrm{P}<0.05)$, and the level of IL-10 increased $(\mathrm{P}<0.05)$. The addition of MSC-EXOs to LPS-induced RAW264.7 cells promoted their polarization toward the M2 phenotype and upregulated their marker expression levels $(\mathrm{P}<0.05)$. Following inhibition of miR-21-5p in MSC cells, the EXOs were collected, and it was found that MSC-EXOs that inhibited the expression of miR-21-5p promoted LPS-induced polarization of RAW264.7 cells to the M1 phenotype and upregulated inflammation in the culture supernatant. Furthermore, transfection with miR-21-5p mimics promoted the polarization of RAW264.7 cells to the M2 phenotype and reduced the level of inflammatory factors in the culture supernatant.

Conclusions: MSC-EXOs promote the polarization of macrophages to the M2 phenotype via miR-21-5p, thereby reducing inflammation and promoting heart repair.

Keywords: Mesenchymal stem cells (MSCs); exosomes; macrophages; polarization; miRNA

Submitted Jun 17, 2021. Accepted for publication Aug 05, 2021.

doi: $10.21037 / \mathrm{atm}-21-3557$

View this article at: https://dx.doi.org/10.21037/atm-21-3557 


\section{Introduction}

Acute myocardial infarction (AMI) is myocardial necrosis caused by acute and persistent ischemia and hypoxia of the coronary artery. It is one of the leading causes of death and disability worldwide (1). Myocardial reperfusion can be achieved within 1 hour after AMI and significantly reduces myocardial injury and infarct size. However, it can also cause further damage to the myocardial tissue during reperfusion, which is called myocardial ischemia-reperfusion injury (2). Myocardial ischemiareperfusion injury activates the inflammatory reaction of myocardial tissue, and the severity and duration of this inflammatory reaction are closely related to the degree of myocardial injury, in which macrophages play an essential role $(3,4)$. Macrophages can polarize into classical activated macrophages (M1 macrophages) or alternative activated macrophages (M2 macrophages) in different environments. M1 macrophages and M2 macrophages have almost antagonist functions (5). M1 macrophages kill microorganisms and tumor cells and secrete a large number of pro-inflammatory factors, whereas M2 macrophages secrete various anti-inflammatory factors and secrete multiple growth factors, which play a crucial role in wound healing and tissue repair (6).

The mesenchymal stem cell (MSC) is a multi-functional stem cell, which has all the typical characteristics of stem cells (7). Recent studies have found that MSCs play a role in reducing myocardial infarction, improving cardiac function, and regulating the inflammatory response $(8,9)$. As reported by Philipp et al., MSCs regulate the level of pro-inflammatory factors produced by macrophages in vitro and in vivo and induce macrophages to polarize to the M2 phenotype (10). Other studies have reported that exosomes (EXOs) mediate the protective effect of MSCs on cardiomyocytes rather than by differentiating them into cardiomyocytes (11). EXOs contain microRNAs (miRNAs) and proteins, which are key components in MSCs (12). Xiao et al. have shown that mesenchymal stem cell-derived exosomes (MSC-EXOs) can induce an anti-inflammatory environment in mice with myocardial ischemia-reperfusion injury (13). However, the specific mechanism of MSCEXOs remains unclear, so this study aims to further explore the protective effect of MSC-EXOs on myocardial ischemia-reperfusion injury and their specific mode of action. We present the following article in accordance with the ARRIVE reporting checklist (available at https://dx.doi. org/10.21037/atm-21-3557).

\section{Methods}

\section{Materials}

The C57BL/6 mouse MSC cell line (article no. MUBMX-01001) was purchased from Suzhou Saiye Biological Co., Ltd., and the RAW264.7 cell line (product no. MZ-2039) was purchased from Ningbo Mingzhou Biotechnology Co., Ltd. Nine 8-week-old male C57BL/6 mice were purchased from the Model Animal Research Center of Nanjing University. Experiments were performed under a project license (No.: SCXK 2016-0009) granted by the Experimental Animals Ethics Committee of Renji Hospital, in compliance with national and Renji Hospital guidelines for the care and use of animals.

Fetal bovine serum, penicillin, streptomycin, Dulbecco's Modified Eagle Medium (DMEM), trypsin, and phosphate buffer saline (PBS) were purchased from China Semer Fisher Technology Co., Ltd. The Lipofectamine 2000 transfection kit and TRIzol kit were purchased from Invitrogen (Carlsbad, California, USA). The exosome extraction kit was purchased from Shanghai Beibo Biological Co., Ltd. Isoflurane was purchased from China Hayward Life Technology Co., Ltd. The Prime ScriptTM RT Master Mixture kit and SYBR Prime Script RT-PCR kit were purchased from Takara, Japan. The RIPA lysis buffer, BCA kit, and ECL luminescent reagent box were purchased from Shanghai Ruji Biotechnology Co., Ltd. The antibody against CD9, CD63, TSG101, Alix, GAPDH, and polyclonal peroxidase-labeled Goat anti-rabbit IgG was purchased from Abcam, UK. The ELISA kits were purchased from R\&O systems, USA. The miR-21-5p inhibitor and miR-21-5p mimics were designed and synthesized by Shandong Weizhen Biotechnology Co., Ltd.

\section{Cell culture and transfection}

The MSC and RAW264.7 cells were cultured in DMEM supplemented with $10 \%$ fetal bovine serum, $100 \mu \mathrm{g} / \mathrm{mL}$ penicillin, and $100 \mu \mathrm{g} / \mathrm{mL}$ streptomycin. All cells were cultured at $37^{\circ} \mathrm{C}$ in a $5 \% \mathrm{CO}_{2}$ environment. The cells were collected in the logarithmic growth phase. After trypsin digestion, the cell density was adjusted to $1 \times 10^{5}$ cells $/ \mathrm{mL}$. Subsequently, $2 \mathrm{~mL}$ of cell suspension was added into each well of the 6-well plate, and the culture was grown at $37{ }^{\circ} \mathrm{C}$ in a $5 \% \mathrm{CO}_{2}$ environment for $24 \mathrm{~h}$. The miR-21-5p inhibitor and miR-21-5p mimics were transfected into the MSC and RAW264.7 cells according to the instructions 
in the Lipofectamine 2000 transfection kit. After $48 \mathrm{~h}$ of transfection, RT-qPCR was performed to examine the results of the transfection.

\section{Isolation of MSC-EXOs}

When the MSCs fused to $80 \%$, they were cultured and replaced with the medium without exosome serum. The culture supernatant of the third generation MSCs was collected and purified by a $0.22 \mu \mathrm{m}$ filter and concentrated according to the instructions of the exosome extraction kit. After mixing, the supernatant was incubated overnight at $4{ }^{\circ} \mathrm{C}$. After centrifuging at $4{ }^{\circ} \mathrm{C}(10,000 \times \mathrm{g})$ for $1 \mathrm{~h}$ and removing the supernatant, the exosome pellet was obtained.

\section{Construction of the myocardial ischemia-reperfusion injury model in mice}

Six C57BL/6 mice were anesthetized with isoflurane and ventilated by a rodent ventilator. The thoracic cavity was opened around the fourth rib space, and the left anterior descending coronary artery was ligated. After $45 \mathrm{~min}$, the ligation was stopped for reperfusion to construct the animal model of myocardial ischemia-reperfusion injury. The mice were randomly allocated to two groups, an MSC-EXO group and a PBS group. Mice in the MSC-EXO group ( $\mathrm{n}=3$ ) were injected with $25 \mu \mathrm{L}$ PBS and $50 \mu \mathrm{g}$ MSC-EXO into three myocardial tissue regions. Mice in the PBS group $(n=3)$ were injected with $25 \mu \mathrm{L}$ of PBS into three myocardial tissue regions. A further three C57BL/6 mice were allocated as the control group and underwent thoracic surgery after anesthesia, but the left anterior descending coronary artery of this group was not ligated. All mice were kept in a laboratory with a suitable temperature and humidity and had free access to food and water under a 12-h dark/light phase condition.

\section{Measurement of the left ventricular ejection fraction $(\mathrm{EF})$ and left ventricular fractional shortening (FS) in mice}

The mice were anesthetized with isoflurane and fixed on the thermostatic plate of the super high-frequency system. An ultrasonic diagnostic instrument for small animals was used with a central frequency of $30 \mathrm{MHz}$ for ultrasonic examination. The EF and FS of mice were measured by Vevo software developed by VisualSonics in Toronto, Canada.

\section{Real-time fluorescent quantitative PCR (RT-qPCR) detection of the levels of miRNA and macrophage polarization marker $m R N A$}

Total RNA was extracted from the tissues or cells according to the TRIzol kit instructions and then reverse transcribed into cDNA using the Prime Script ${ }^{\mathrm{TM}}$ RT Master Mixture kit. The SYBR Prime Script RT-PCR kit was used to detect the miRNA and mRNA expressions in the tissues and cells. The thermal cycle conditions were as follows: predenaturation at $95^{\circ} \mathrm{C}$ for $60 \mathrm{~s}$, denaturation at $95^{\circ} \mathrm{C}$ for $15 \mathrm{~s}$, annealing at $60{ }^{\circ} \mathrm{C}$ for $40 \mathrm{~s}$, extension at $72{ }^{\circ} \mathrm{C}$ for $15 \mathrm{~s}$, for a total of 40 cycles. The $2^{-\Delta \Delta C T}$ calculation was used to detect the expression levels of miR-21-5p and its related mRNA with internal reference to U6 or GAPDH. The primer sequence is shown in Table 1.

\section{Detection of exosome marker expressions by western blotting}

RIPA lysis buffer was used to extract the total protein from the cells, and a BCA kit was used to determine the protein concentration. The protein was separated by $10 \%$ SDSPAGE and then transferred to a PVDF membrane. The protein was blocked with $5 \%$ skimmed milk powder at room temperature for $2 \mathrm{~h}$ and incubated with anti-CD9 $(1: 1,000)$, anti-CD63 (1:1,000), anti-TSG101 (1:1,000), anti-Alix $(1: 1,000)$, and anti-GAPDH $(1: 2,000)$ at $4{ }^{\circ} \mathrm{C}$ overnight. After washing three times with PBS, the membrane was incubated with a polyclonal peroxidase-labeled second antibody (Goat anti-rabbit IgG with a concentration of $1: 4,000)$ at room temperature for $2 \mathrm{~h}$. Images were obtained with the ECL kit, and ImageJ software was used for the quantitative analysis.

\section{ELISA measurement of IL-6 and IL-10 levels}

The cell culture medium and treated tissue samples were collected and centrifuged $(1,000 \times \mathrm{g})$ for $5 \mathrm{~min}$. After the centrifugation, the cell-free supernatant was obtained. The solution was blocked by PBS for $1 \mathrm{~h}$ and was added to $100 \mu \mathrm{L}$ of supernatant followed by $2 \mathrm{~h}$ of incubation at room temperature. Subsequently, $100 \mu \mathrm{L}$ anti-mouse IgG antibody was added and incubated at room temperature for a further $2 \mathrm{~h}$. Next, $100 \mu \mathrm{L}$ of TMB substrate was added, and the reaction was terminated after 30 min of dark color development at room temperature. The enzyme reader determined the $\mathrm{OD}$ value of the sample at $450 \mathrm{~nm}$, and the 
Table 1 Prime sequence

\begin{tabular}{|c|c|}
\hline Gene & Sequence $\left(5^{\prime}-3^{\prime}\right)$ \\
\hline \multirow[t]{2}{*}{$m i R-21-5 p$} & F: GGGGTAGCTTATCAGACTGATG \\
\hline & R: TGTCGTGGAGCGGCAATTG \\
\hline \multirow[t]{2}{*}{ U6 } & F: CGCTTCGGCACATATACTA \\
\hline & R: CGCTTCACGAATTTGCGTGTCA \\
\hline \multirow[t]{2}{*}{ iNOS } & F: CCAAGAACGTGTTCACCATG \\
\hline & R: GATGTCCAGGAAGTAGGTGAGG \\
\hline \multirow[t]{2}{*}{$\mathrm{IL}-1 \beta$} & F: CTCCATGAGCTTTGTACAAGG \\
\hline & R: TGCTGATGTACCAGTTGGGG \\
\hline \multirow[t]{2}{*}{ IL-6 } & F: GACTGATGTTGTTGACAGCCACTGC \\
\hline & R: AGCCACTCCTTCTGTGACTCTAАCT \\
\hline \multirow[t]{2}{*}{$\mathrm{TNF} \alpha$} & F: CATGATCCGAGATGTGGAACTGGC \\
\hline & R: CTGG6CTCAGCCACTCCAGC \\
\hline \multirow[t]{2}{*}{ Arg1 } & F: GGGAAGACACCAGAAGAAGTAA \\
\hline & R: GTGGGTTAAGGTAGTCAATAGG \\
\hline \multirow[t]{2}{*}{ IL-10 } & F: TGCCAAGCCTTGTCAGAAATGATCAAG \\
\hline & R: GTATCCAGAGGGTCTTCAGCTTCTCTC \\
\hline \multirow[t]{2}{*}{ CD206 } & F: GCAAAGGACAAGAAAGAAGACC \\
\hline & R: TTTCTCCCTTGGGTAGGTGC \\
\hline \multirow[t]{2}{*}{ TGF $\beta$} & F: AACTACTGCTTCAGCTCCAC \\
\hline & R: GTAACACGATGGCGAGTGCG \\
\hline \multirow[t]{2}{*}{ GAPDH } & F: TGGCAAAGTGGAGATTGTTGCC \\
\hline & R: AAGATGGTGATGGGCTTCCCG \\
\hline
\end{tabular}

sample concentration was calculated.

\section{Localization of exosomes in macrophages by immunofluorescence assay}

The slides were washed three times with PBS, then fixed with $4 \%$ paraformaldehyde for $15 \mathrm{~min}$. The slides were rewashed a further three times with PBS and then penetrated with $0.5 \%$ Triton $\mathrm{X}-100$ at room temperature for $20 \mathrm{~min}$. After washing, the goat serum was dripped onto the slide and blocked at room temperature for $30 \mathrm{~min}$. After adding DiI-labeled MSC-EXOs, the cells were blocked overnight at $4{ }^{\circ} \mathrm{C}$. DAPI was added and incubated in the dark for 5 min then washed three times with PBS. Then the sealing solution with a fluorescence quenching agent was used to seal the film. The film was observed and photographed under a fluorescence microscope.

Flow cytometry detection of the proportion of macrophages with M1 and M2 phenotypes

RAW264.7 cells were collected into 96-well plates and adjusted to $5 \times 10^{5} /$ well. They were resuspended in $100 \mu \mathrm{L}$ of PBS, followed by the addition of mouse CD26 antibody and mouse iNOS antibody and incubated at room temperature in the dark for $30 \mathrm{~min}$. The cells were then washed three times with PBS and resuspended in $200 \mu \mathrm{L}$ of PBS. The proportion of macrophages was detected and analyzed by FlowJo software developed by Stanford University.

\section{Statistical analysis}

SPSS 20.0 software developed by SPSS Company (Chicago, Illinois, USA) was used for the statistical analysis. All experiments were repeated five times, and the measurement data were based on the mean \pm standard deviation $(\bar{x} \pm$ $s)$. T-tests were used to compare the data between two groups, and one-way ANOVA was used to compare the data between three groups. $\mathrm{P}<0.05$ indicated a statistically significant difference.

\section{Results}

\section{MSC-EXOs attenuated myocardial injury and inflammation in model mice}

Western blotting was used to detect the exosome markers isolated from the culture supernatant of MSCs. The results showed that CD63, CD9, TSG101, and Alix were expressed in the exosomes, as shown in Figure 1A. MSCEXOs or PBS buffer were injected into the myocardial region of the model mice. The results indicated that, 3 days after surgery, the EF and FS scores of the model mice injected with MSC-EXOs were significantly higher than those of the model mice injected with PBS $(\mathrm{P}<0.05)$. The myocardial tissue remained protected two weeks after surgery $(\mathrm{P}<0.01)$ (Figure $1 B, 1 C)$. Comparison of the infarction of the myocardial tissue of the model mice 3 days after surgery showed that the injection of MSCEXOs into myocardial tissue significantly reduced the infarct size in the model mice $(\mathrm{P}<0.01)$ (Figure $1 D)$. In addition, compared with the control group, the expression levels of IL-6 and IL-10 in the serum and myocardial 

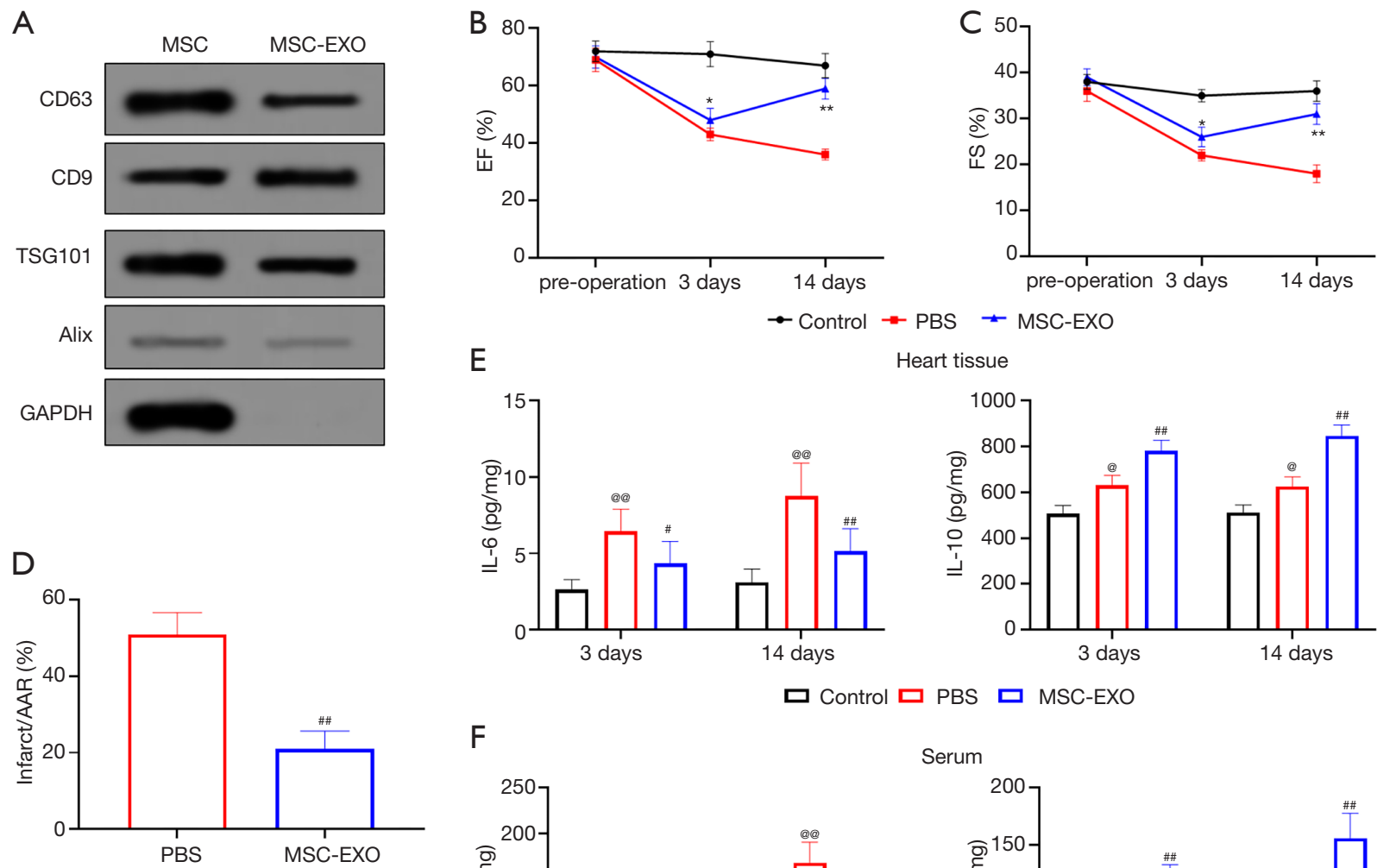

Heart tissue
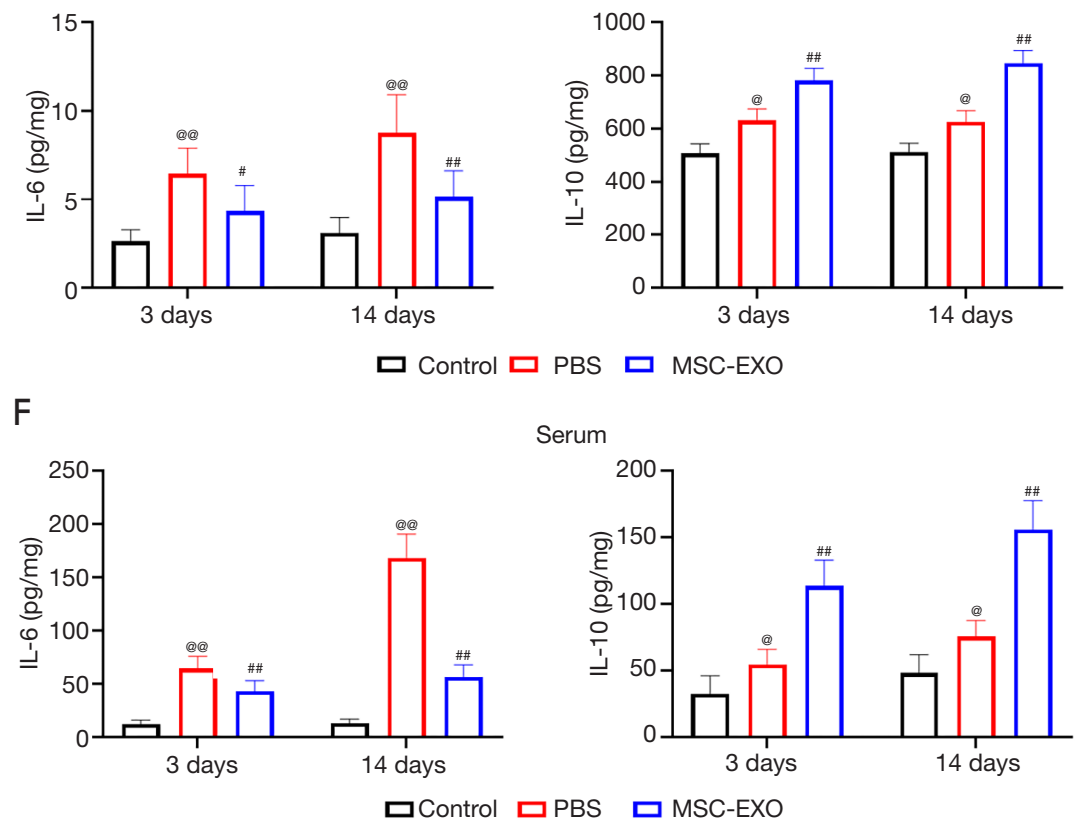

Figure 1 MSC-EXOs can reduce myocardial injury and inflammation in mice. (A) The expression levels of protein markers of MSC-EXOs; (B,C) EF and FS scores of mice at different times after surgery; (D) the ratio of the myocardial infarct area 3 days after surgery. (E,F) IL-6 and IL-10 levels in the serum or heart tissues of mice at different times after surgery. ${ }^{*} \mathrm{P}<0.05,{ }^{* *} \mathrm{P}<0.01$ vs. pre-operation group; ${ }^{\#} \mathrm{P}<0.05$ vs. PBS group; ${ }^{\#} \mathrm{P}<0.01$ vs. PBS group; ${ }^{\circledR \oplus} \mathrm{P}<0.01$ vs. Control group

tissues of the model mice were significantly increased $(\mathrm{P}<0.05)$. Compared with the model mice injected with PBS buffer, the level of IL-6 in the serum and tissue of the model mice injected with MSC-EXOs was significantly decreased $(\mathrm{P}<0.05)$, and the level of IL-10 was significantly increased $(\mathrm{P}<0.01)$, as shown in Figure $1 E, 1 F$.

\section{MSC-EXOs promoted macrophage polarization to the M2 phenotype in model mice}

Macrophages can switch between M1 and M2 phenotypes when subjected to external stimulation. RT-qPCR results indicated the mRNA levels of the M1 macrophage markers
(iNOS, IL-1 $\beta$, IL-6, and TNF $\alpha$ ) in the myocardial tissue were significantly reduced $(\mathrm{P}<0.05) 3$ days after injection with MSC-EXOs. In contrast, the mRNA expression levels of M2 macrophage markers (Arg1, IL-10, CD206, and TGF $\beta$ ) were significantly increased $(\mathrm{P}<0.05)$ compared with mice injected with PBS (Figure 2).

\section{MSC-EXOs promoted the conversion of macrophages to the M2 phenotype}

Lipopolysaccharide (LPS) was added to the culture medium and co-cultured with RAW264.7 cells to induce the inflammatory microenvironment. The ELISA results 

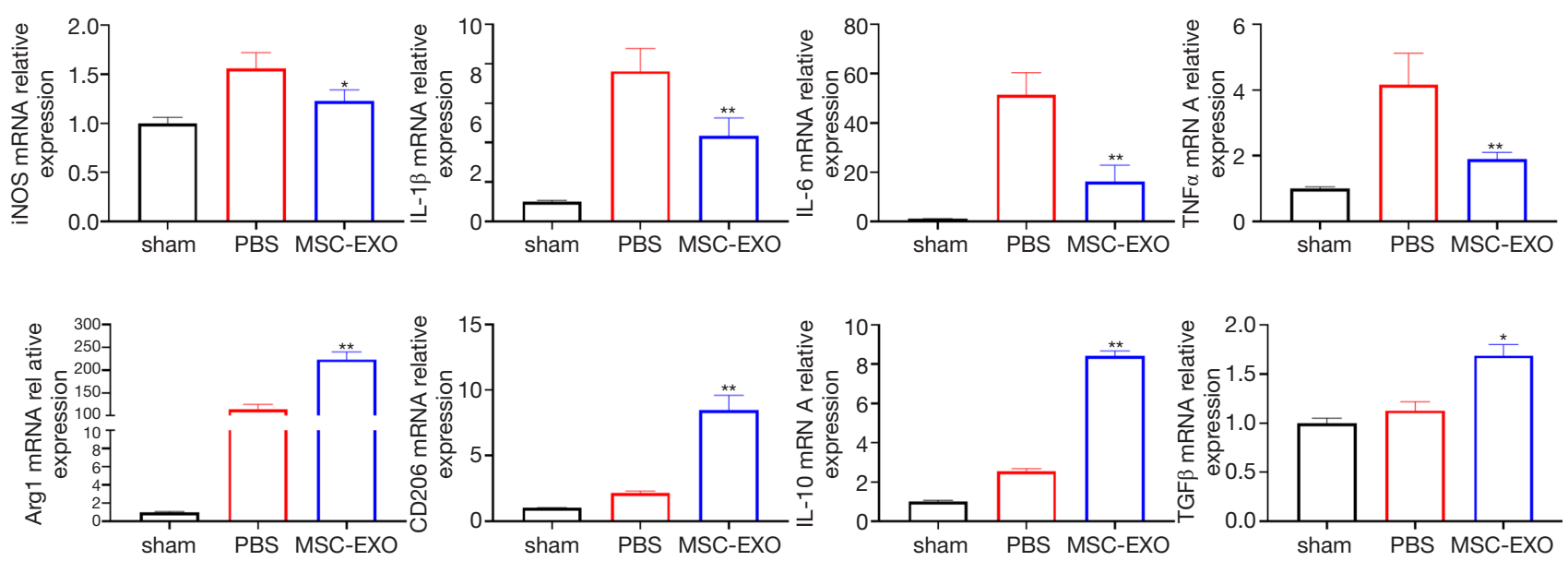

Figure 2 MSC-EXOs promote the polarization of macrophages to the M2 phenotype in mice. The expression of M1 or M2 macrophage markers in the myocardium of mice 3 days after surgery. ${ }^{*} \mathrm{P}<0.05,{ }^{* *} \mathrm{P}<0.01$ vs. $\mathrm{PBS}$ group.

showed that LPS induction significantly increased the levels of IL-6 and IL-10 in the culture supernatant $(\mathrm{P}<0.05)$. After adding MSC-EXOs, it significantly reduced the level of IL- 6 in the medium and increased the level of IL-10 $(\mathrm{P}<0.05)$, as shown in Figure 3A. MSCEXOs were co-cultured with RAW264.7 cells, and DiIlabeled MSC-EXOs were evenly distributed in the cytoplasm of RAW264.7 cells, as shown in Figure 3B. The results of flow cytometry experiments showed that after adding MSC-EXOs, the proportion of M2 macrophages was significantly increased $(\mathrm{P}<0.01)$, and the proportion of M1 macrophages was significantly reduced $(\mathrm{P}<0.01)$, as shown in Figure $3 C$. The M1 and M2 macrophage markers were detected by RT-qPCR. After adding MSCEXOs, the mRNA levels of the M1 macrophage markers (iNOS, IL-1 $\beta$, IL-6, and TNF $\alpha$ ) were significantly suppressed $(\mathrm{P}<0.01)$, whereas the mRNA levels of the M2 macrophage markers (Arg1, IL-10, CD206, and TGF $\beta$ ) were significantly increased $(\mathrm{P}<0.05)$ (Figure $3 D)$.

\section{miR-21-5p inbibited the polarization of macrophages to the M1 phenotype}

RT-qPCR results showed that the injection of MSC-EXOs into the myocardial tissue of model mice significantly upregulated the expression of miR-21-5p in the myocardial tissue $(\mathrm{P}<0.01)$. Similarly, adding MSC-EXOs to the culture medium also significantly upregulated the expression level of miR-21-5p in RAW264.7 cells $(\mathrm{P}<0.01)$ (Figure $4 A$ ). An miR-21-5p inhibitor was transfected into MSCs to clarify the role of miR-21-5p. RT-qPCR results showed that EXOs in the MSCs transfected with the miR-21-5p inhibitor had a significantly reduced expression of miR-21$5 \mathrm{p}(\mathrm{P}<0.01)$, as shown in Figure 4B. MSC-EXOs inhibiting miR-21-5p were used to treat LPS-induced RAW264.7 cells for $48 \mathrm{~h}$. The results of flow cytometry and RT-qPCR experiments indicated that MSC-EXOs inhibiting miR-21$5 \mathrm{p}$ significantly reduced the conversion of macrophages to the M2 phenotype and upregulated M1 macrophage marker expressions in RAW264.7 cells $(\mathrm{P}<0.05)$ (Figure $4 C, 4 D)$. At the same time, adding MSC-EXOs that inhibited miR-21$5 p$ caused upregulation of the level of IL-6 in the medium supernatant $(\mathrm{P}<0.01)$ and downregulation of the level of IL$10(\mathrm{P}<0.01)$, as shown in Figure $4 E$. To further verify the role of miR-21-5p in macrophage transformation to the M2 phenotype, miR-21-5p mimics were directly transfected into RAW264.7 cells. RT-qPCR results showed that the level of miR-21-5p in the RAW264.7 cells was significantly increased after transfection with miR-21-5p mimics $(\mathrm{P}<0.01)$, as shown in Figure $4 F$. The effect of miR-21-5p on the polarization of the RAW264.7 cells was detected by flow cytometry. The results showed that overexpression of miR-21-5p significantly increased the proportion of M2 macrophages. In contrast, the mRNA levels of the M1 macrophage markers in the RAW264.7 cells (iNOS, IL-1 $\beta$, IL-6, and TNF $\alpha$ ) were significantly reduced $(\mathrm{P}<0.01)$. The mRNA levels of the M2 macrophage markers (Arg1, IL-10, CD206, and TGF $\beta$ were significantly increased $(\mathrm{P}<0.05)$ (Figure $4 G, 4 H$ ). At the same time, overexpression of miR21-5p significantly reduced the level of IL-6 in the culture 
A

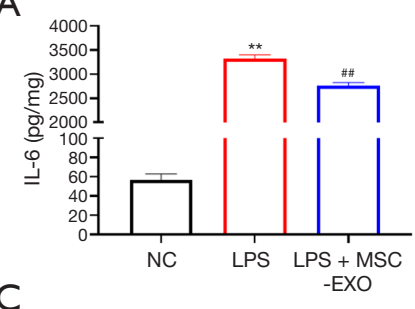

C
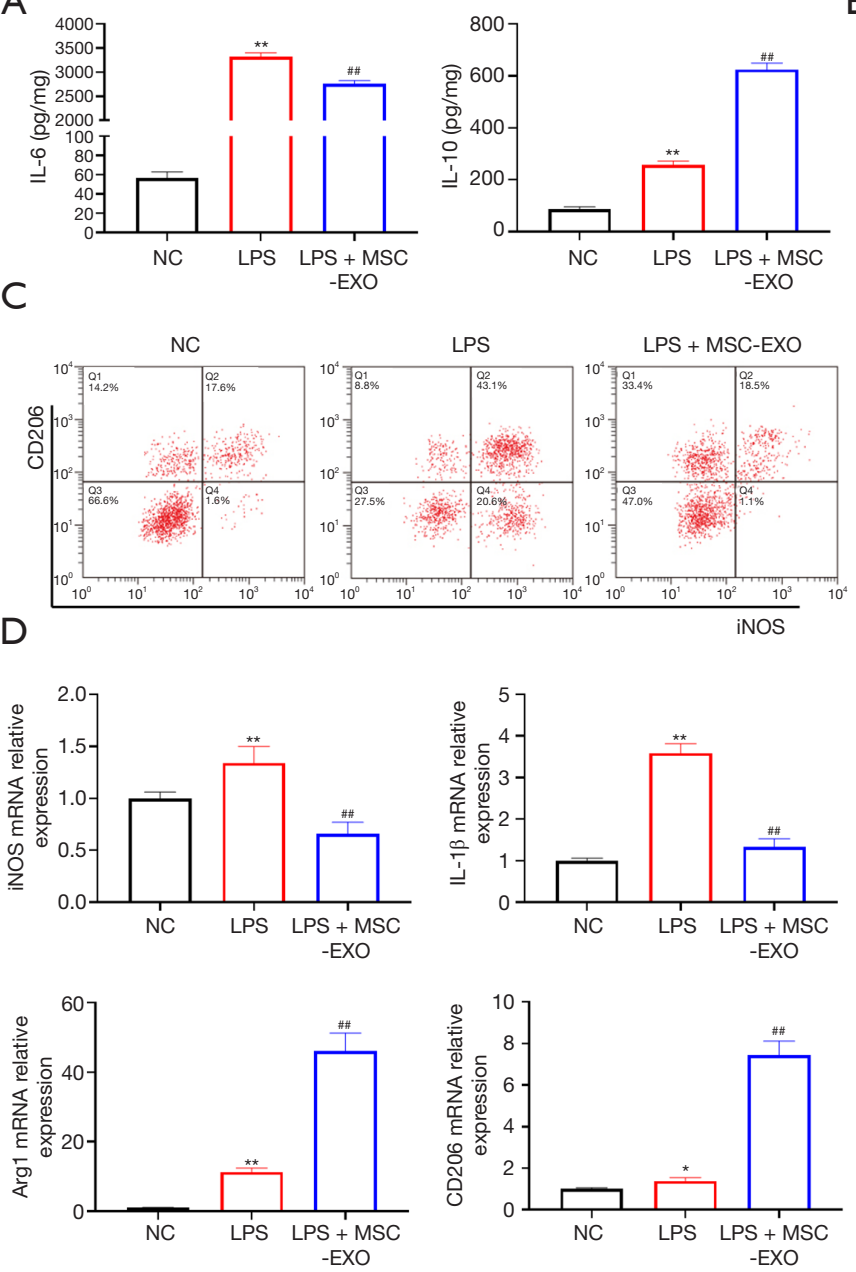

B
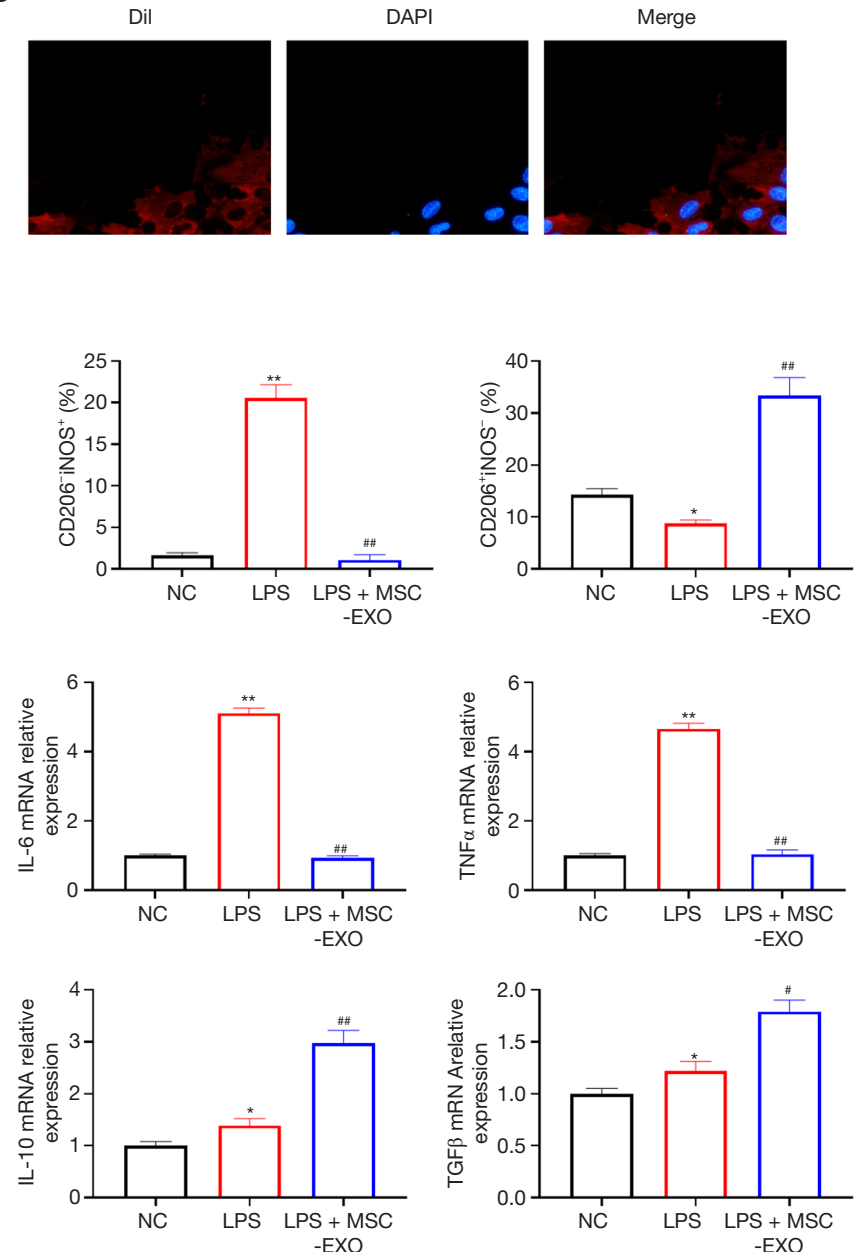

Figure 3 MSC-EXOs promote the transformation of macrophages into the M2 phenotype. (A) Levels of IL-6 and IL-10 in the culture medium supernatant; (B) localization of MSC-EXOs by immunofluorescence staining method in RAW264.7 cells (magnification, 100×); (C) the proportion of M1 to M2 macrophages in RAW264.7 cells in each group; (D) the expression levels of M1 or M2 macrophage markers in RAW264.7 cells in each group. ${ }^{*} \mathrm{P}<0.05,{ }^{* *} \mathrm{P}<0.01$ vs. NC group; ${ }^{*} \mathrm{P}<0.05,{ }^{\# \#} \mathrm{P}<0.01$ vs. LPS group.

supernatant of the RAW264.7 cells and increased IL-10 $(\mathrm{P}<0.01)$, as shown in Figure 41 .

\section{Discussion}

After myocardial ischemia-reperfusion injury, necrotic cardiomyocytes release various cytokines to induce an inflammatory response (14). In the early stage of myocardial ischemia, the inflammatory reaction removes necrotic myocardial cells and debris. However, in this process, the infiltration of inflammatory cells and the inflammatory factors produced by them can also cause further damage to normal myocardial cells (14). The ultimate goal of clinical treatment of AMI is to reduce myocardial injury and restore the cardiac function of patients to the best possible level. However, some drugs found to be effective for myocardial ischemia-reperfusion in animal experiments have not achieved an ideal therapeutic effect in clinical trials (1). Therefore, it is necessary to explore new therapeutic ideas for the clinical treatment of AMI.

Mesenchymal stem cells have multidirectional differentiation potential, differentiate into functional cardiomyocyte-like cells in the microenvironment of myocardial tissue, and form connections with host cells to promote the repair of myocardial tissue. At the same time, mesenchymal stem cells can differentiate into 


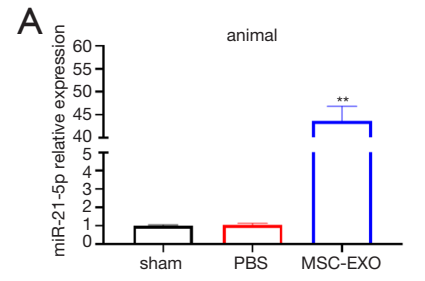

B

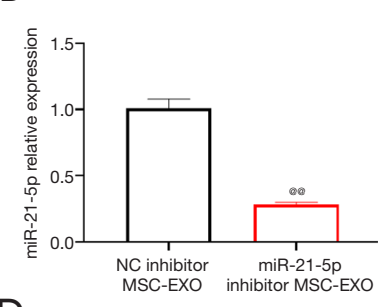

D
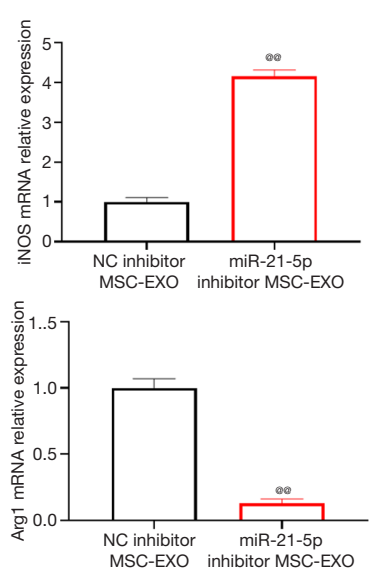

$\mathrm{F}$

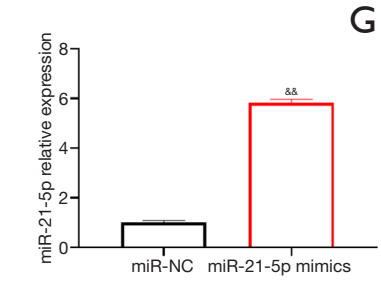

$\mathrm{H}$
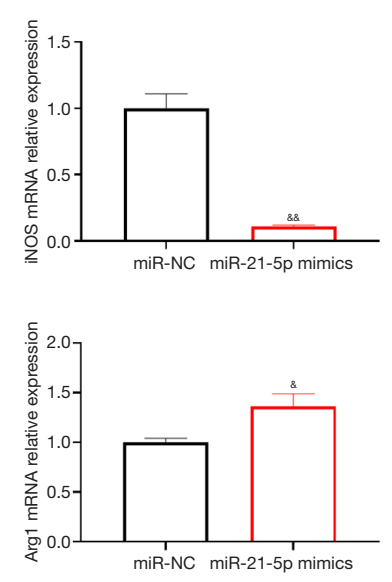
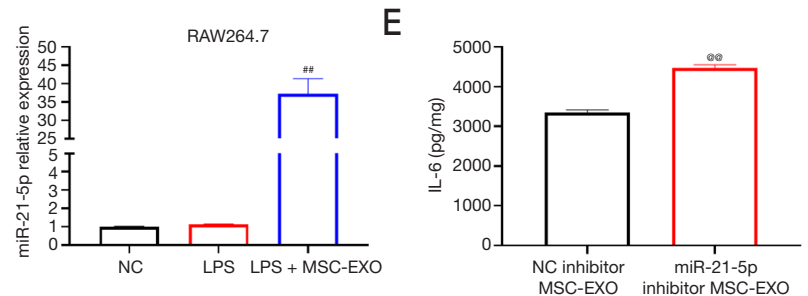

C
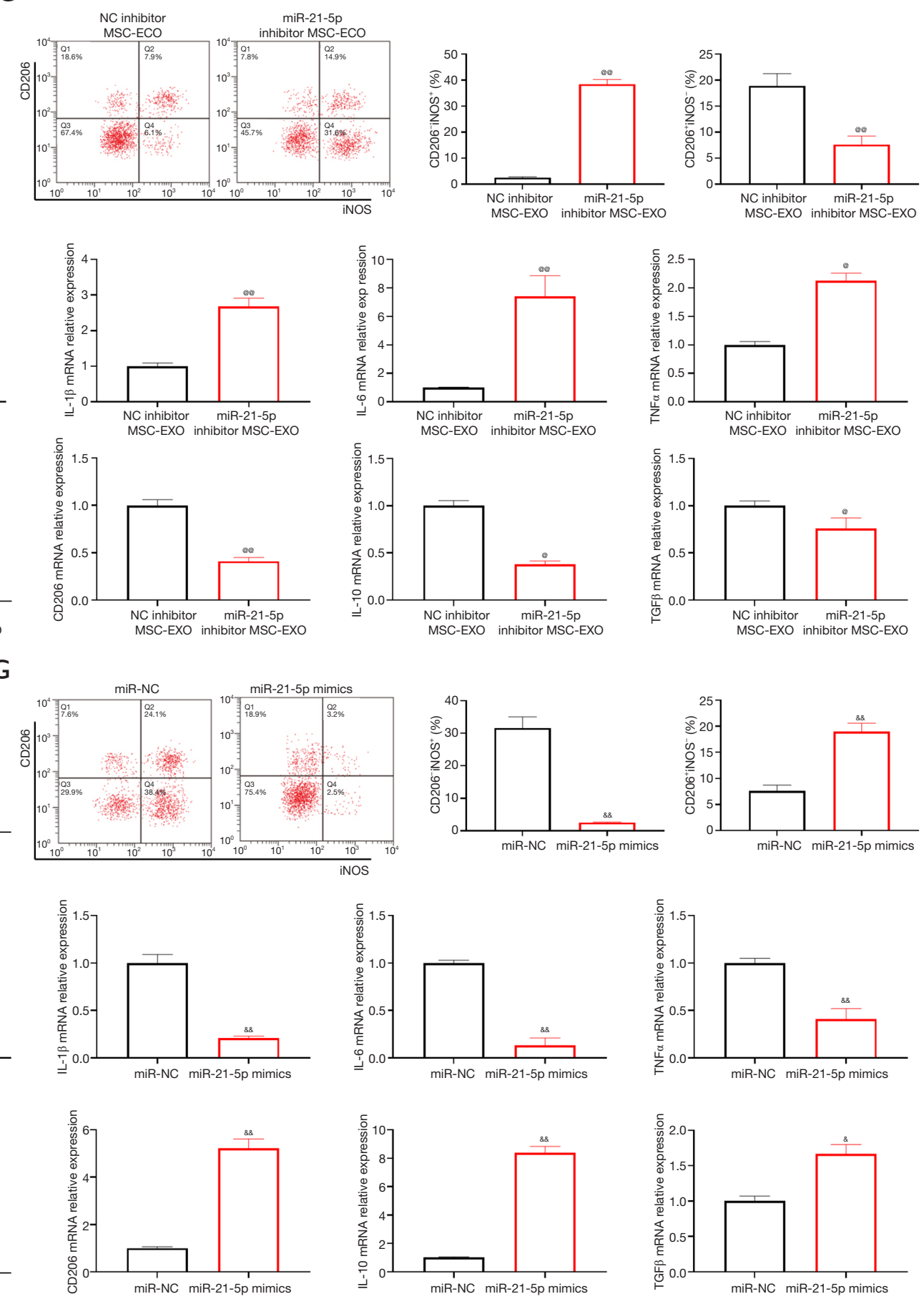

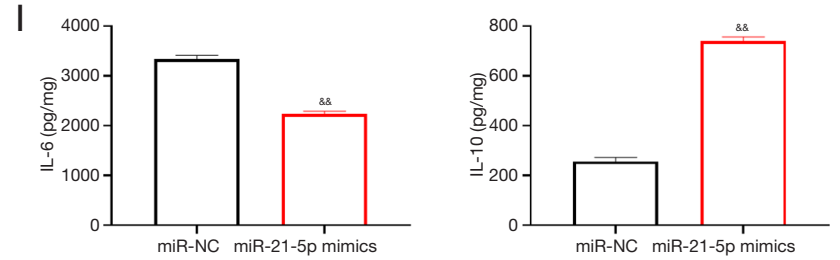

Figure $4 \mathrm{miR}-21-5 \mathrm{p}$ is a key regulator of the inhibition of macrophage polarization to the M1-type phenotype. (A) The expression levels of miR-21-5p in C57BL/6 mice and RAW264.7 cells; (B,F) The expression levels of miR-21-5p in MSC-EXOs and RAW264.7 cells; (C,G) the proportion of M1 to M2 macrophages in RAW264.7 cells in each group; (D,H) the expression levels of M1 or M2 macrophage markers in RAW264.7 cells in each group; (E,I) the levels of IL-6 and IL-10 in the culture medium supernatant. ${ }^{* *} \mathrm{P}<0.01$ vs. $\mathrm{PBS}$ group; ${ }^{\# \#} \mathrm{P}<0.01$ vs. LPS group; ${ }^{\circledR} \mathrm{P}<0.05,{ }^{\circledR} \mathrm{P}<0.01$ vs. NC inhibitor MSC-EXO group; ${ }^{\circledR} \mathrm{P}<0.05,{ }^{\& \&} \mathrm{P}<0.01$ vs. NC inhibitor MSC-EXO group.

vascular endothelial cells and vascular smooth muscle cells in the ischemic myocardial blood vessels, which can effectively promote cardiovascular formation, improve the blood supply of local myocardial tissues, and save dying myocardial cells. Mesenchymal stem cells can also secrete a variety of cytokines, promote the proliferation and differentiation of cardiomyocytes, and are beneficial to the regeneration of cardiomyocytes and blood vessels. In the past decade, it has been found that MSCs can interact with various cells in the immune system, which provides the possibility for treatment of a variety of immune-related diseases (15). Some animal model studies have shown that MSCs can promote repair in myocardial injury (8). At present, it is generally believed that the interaction between MSCs and the inflammatory microenvironment depends on EXOs to a great extent (16). EXOs are tiny vesicles secreted by various cells, improving heart function and reducing ventricular remodeling by inhibiting apoptosis, reducing oxidative stress, and stimulating angiogenesis (11). In this study, we found that direct injection of MSC-EXOs into the myocardial tissue of mice with myocardial ischemiareperfusion injury significantly improved the EF and FS scores and reduced the infarct size. MSC-EXOs showed continued protection of the myocardial tissue two weeks after injection. After injection of MSC-EXOs, the level of IL-6 in the serum and myocardial tissue of model mice decreased, while the level of IL-10 increased. In addition, injection of MSC-EXOs significantly reduced the level of M1 macrophage markers in the myocardial tissue of mice and increased the level of M2 macrophage markers. These results suggest that injection of MSC-EXOs can promote the recovery of cardiac function and reduce inflammatory reaction in the early and recovery stages of myocardial ischemia-reperfusion injury. This protective effect may be achieved by reducing the proportion of M1 macrophages.
Macrophages are the primary mediators of inflammation after myocardial ischemia-reperfusion injury. Many studies have shown that macrophages play an essential role in myocardial repair after myocardial ischemia-reperfusion injury $(4,17,18)$. Currently, it is generally believed that EXOs secreted by MSCs realize the interaction between MSCs and macrophages. This study found that MSC-EXOs were evenly distributed in the cytoplasm of macrophages after co-culture with macrophages. An increasing number of studies have shown that MSCs can induce macrophages to switch to the M2 phenotype to reduce the inflammatory response $(19,20)$. In this study, LPS was added to the medium of RAW264.7 cells to simulate the inflammatory microenvironment. It was found that MSC-EXOs significantly reduced IL-6 levels in the medium supernatant and significantly increased the level of IL-10. In addition, MSC-EXOs successfully transformed the polarization state of RAW264.7 macrophages from the M1 phenotype to the M2 phenotype in vitro. These results suggest that MSCEXOs can promote macrophage polarization to the M2 phenotype in vitro and reduce the inflammatory response.

miRNA is a crucial component of EXOs and determines the effect of EXOs on receptor cells. Some miRNAs found in MSC-EXOs play key roles in immune regulation, such as miR-125a (21), miR-146a (16), miR-181b (22), miR223 (23), and miR-21-5p (24). In previous experiments, we found that the expression level of miR-21-5p in MSCEXOs was higher than that in macrophage-derived exosomes. In this study, we found that MSC-EXOs significantly increased the level of miR-21-5p in myocardial tissue and LPS-induced RAW264.7 cells of model mice, suggesting that miR-21-5p may be involved in the process of macrophage polarization to the M2 phenotype and in alleviating the inflammatory response. MiR-21-5p, located on chromosome $17 \mathrm{q} 23.1$, is an anti-apoptotic gene. 
Previous studies have shown that miR-21-5p can protect cells from oxidative stress-induced cell death by mediating MSC-EXOs (25). Li et al. showed that MSC-EXOs could effectively reduce lung ischemia-reperfusion injury in mice via miR-21-5p (26). The present study found that overexpression of miR-21-5p attenuated the LPS-induced inflammatory response of RAW264.7 cells and promoted the M2 phenotype transformation of RAW264.7 cells. In addition, the inhibition of miR-21-5p expression in MSCEXOs weakened the immunomodulatory effect of MSCEXOs on macrophages. That is, miR-21-5p participated in the inflammatory response and MSC-EXOs mediated macrophage polarization. Although this study confirmed that MSC-EXOs participate in the immune process of macrophages via miR-21-5p, its specific mechanism is still unclear. Previous studies have found that toll-like receptor 4 (TLR4) is the downstream target gene of mir-21-5p. Yang et al. showed that TLR4 promoted the expression of inflammatory factors in various cardiovascular diseases (27). Similarly, Hua et al. showed that TLR4 also regulated the activation of the PI3K/Akt signaling pathway (28). In addition, studies have shown that the PI3K/Akt signaling pathway is critical in the transformation of macrophages into the M2 phenotype (29). Therefore, we have reason to believe that the miR-21-5p/TLR4/PI3K/Akt signaling pathway may be an important pathway involved in the immune process of macrophages, but whether this pathway really plays a role still needs to be further explored.

Although there is strong evidence that miR-21$5 \mathrm{p}$ plays a crucial role in promoting cardiac repair and alleviating inflammatory response, the contribution of other active substances in MSC-EXOs in this process cannot be excluded. In conclusion, this study confirmed that MSC-EXOs induced macrophages to polarize to the M2 phenotype via miR-21-5p to reduce the inflammatory response and promote cardiac repair, providing theoretical support for MSC-EXOs as a potential therapeutic approach for myocardial ischemia-reperfusion injury.

\section{Acknowledgments}

Funding: None.

\section{Footnote}

Reporting Checklist: The authors have completed the ARRIVE reporting checklist. Available at https://dx.doi. org/10.21037/atm-21-3557
Data Sharing Statement: Available at https://dx.doi. org/10.21037/atm-21-3557

Conflicts of Interest: Both authors have completed the ICMJE uniform disclosure form (available at https://dx.doi. org/10.21037/atm-21-3557). The authors have no conflicts of interest to declare.

Etbical Statement: The authors are accountable for all aspects of the work in ensuring that questions related to the accuracy or integrity of any part of the work are appropriately investigated and resolved. Experiments were performed under a project license (No.: SCXK 2016-0009) granted by the Experimental Animals Ethics Committee of Renji Hospital, in compliance with national and Renji Hospital guidelines for the care and use of animals.

Open Access Statement: This is an Open Access article distributed in accordance with the Creative Commons Attribution-NonCommercial-NoDerivs 4.0 International License (CC BY-NC-ND 4.0), which permits the noncommercial replication and distribution of the article with the strict proviso that no changes or edits are made and the original work is properly cited (including links to both the formal publication through the relevant DOI and the license). See: https://creativecommons.org/licenses/by-ncnd/4.0/.

\section{References}

1. Shah AH, Puri R, Kalra A. Management of cardiogenic shock complicating acute myocardial infarction: A review. Clin Cardiol 2019;42:484-93.

2. Chi HJ, Chen ML, Yang XC, et al. Progress in Therapies for Myocardial Ischemia Reperfusion Injury. Curr Drug Targets 2017;18:1712-21.

3. Xu Z, McElhanon KE, Beck EX, et al. A Murine Model of Myocardial Ischemia-Reperfusion Injury. Methods Mol Biol 2018;1717:145-53.

4. de Couto G, Liu W, Tseliou E, et al. Macrophages mediate cardioprotective cellular postconditioning in acute myocardial infarction. J Clin Invest 2015;125:3147-62.

5. Artyomov MN, Sergushichev A, Schilling JD. Integrating immunometabolism and macrophage diversity. Semin Immunol 2016;28:417-24.

6. Wang Y, Smith W, Hao D, et al. M1 and M2 macrophage polarization and potentially therapeutic naturally occurring compounds. Int Immunopharmacol 2019;70:459-66. 
7. Uder C, Brückner S, Winkler S, et al. Mammalian MSC from selected species: Features and applications. Cytometry A 2018;93:32-49.

8. Luo L, Tang J, Nishi K, et al. Fabrication of Synthetic Mesenchymal Stem Cells for the Treatment of Acute Myocardial Infarction in Mice. Circ Res 2017;120:1768-75.

9. Harrell CR, Jovicic N, Djonov V, et al. Mesenchymal Stem Cell-Derived Exosomes and Other Extracellular Vesicles as New Remedies in the Therapy of Inflammatory Diseases. Cells 2019;8:1605.

10. Philipp D, Suhr L, Wahlers T, et al. Preconditioning of bone marrow-derived mesenchymal stem cells highly strengthens their potential to promote IL-6-dependent M2b polarization. Stem Cell Res Ther 2018;9:286.

11. Ju C, Shen Y, Ma G, et al. Transplantation of Cardiac Mesenchymal Stem Cell-Derived Exosomes Promotes Repair in Ischemic Myocardium. J Cardiovasc Transl Res 2018;11:420-8.

12. Ju C, Li Y, Shen Y, et al. Transplantation of Cardiac Mesenchymal Stem Cell-Derived Exosomes for Angiogenesis. J Cardiovasc Transl Res 2018;11:429-37.

13. Xiao C, Wang K, Xu Y, et al. Transplanted Mesenchymal Stem Cells Reduce Autophagic Flux in Infarcted Hearts via the Exosomal Transfer of miR-125b. Circ Res 2018;123:564-78.

14. Fröhlich GM, Meier P, White SK, et al. Myocardial reperfusion injury: looking beyond primary PCI. Eur Heart J 2013;34:1714-22.

15. Le Blanc K, Davies LC. Mesenchymal stromal cells and the innate immune response. Immunol Lett 2015;168:140-6.

16. Ti D, Hao H, Fu X, et al. Mesenchymal stem cells-derived exosomal microRNAs contribute to wound inflammation. Sci China Life Sci 2016;59:1305-12.

17. Thackeray JT, Hupe HC, Wang Y, et al. Myocardial Inflammation Predicts Remodeling and Neuroinflammation After Myocardial Infarction. J Am Coll Cardiol 2018;71:263-75.

18. Wang C, Zhang C, Liu L, et al. Macrophage-Derived mir-155-Containing Exosomes Suppress Fibroblast Proliferation and Promote Fibroblast Inflammation during Cardiac Injury. Mol Ther 2017;25:192-204.

19. Willis GR, Fernandez-Gonzalez A, Anastas J, et al. Mesenchymal Stromal Cell Exosomes Ameliorate Experimental Bronchopulmonary Dysplasia and Restore Lung Function through Macrophage Immunomodulation. Am J Respir Crit Care Med 2018;197:104-16.

20. Zhao J, Li X, Hu J, et al. Mesenchymal stromal cell- derived exosomes attenuate myocardial ischaemiareperfusion injury through miR-182-regulated macrophage polarization. Cardiovasc Res 2019;115:1205-16.

21. Liang X, Zhang L, Wang S, et al. Exosomes secreted by mesenchymal stem cells promote endothelial cell angiogenesis by transferring miR-125a. J Cell Sci 2016;129:2182-9.

22. de Couto G, Gallet R, Cambier L, et al. Exosomal MicroRNA Transfer Into Macrophages Mediates Cellular Postconditioning. Circulation 2017;136:200-14.

23. Chen L, Lu FB, Chen DZ, et al. BMSCs-derived miR223-containing exosomes contribute to liver protection in experimental autoimmune hepatitis. Mol Immunol 2018;93:38-46.

24. Wu L, Xia J, Li D, et al. Mechanisms of M2 MacrophageDerived Exosomal Long Non-coding RNA PVT1 in Regulating Th17 Cell Response in Experimental Autoimmune Encephalomyelitisa. Front Immunol 2020;11:1934.

25. Mayourian J, Ceholski DK, Gorski PA, et al. Exosomal microRNA-21-5p Mediates Mesenchymal Stem Cell Paracrine Effects on Human Cardiac Tissue Contractility. Circ Res 2018;122:933-44.

26. Li JW, Wei L, Han Z, et al. Mesenchymal stromal cellsderived exosomes alleviate ischemia/reperfusion injury in mouse lung by transporting anti-apoptotic miR-21-5p. Eur J Pharmacol 2019;852:68-76.

27. Yang Y, Lv J, Jiang S, et al. The emerging role of Toll-like receptor 4 in myocardial inflammation. Cell Death Dis 2016;7:e2234.

28. Hua F, Ha T, Ma J, et al. Protection against myocardial ischemia/reperfusion injury in TLR4-deficient mice is mediated through a phosphoinositide 3-kinase-dependent mechanism. J Immunol 2007;178:7317-24.

29. Li X, Huang Q, Wang M, et al. Compound K Inhibits Autophagy-Mediated Apoptosis Through Activation of the PI3K-Akt Signaling Pathway Thus Protecting Against Ischemia/Reperfusion Injury. Cell Physiol Biochem 2018;47:2589-601.

(English Language Editor: D. Fitzgerald)

Cite this article as: Shen D, He Z. Mesenchymal stem cellderived exosomes regulate the polarization and inflammatory response of macrophages via miR-21-5p to promote repair after myocardial reperfusion injury. Ann Transl Med 2021;9(16):1323. doi: 10.21037/atm-21-3557 